\title{
Retinal Arteriolar Tortuosity and Other Dimorphisms - A New Syndrome
}

\author{
Jessica G Sigler Morales ${ }^{1}$ and Aldo A Sigler Villanueva ${ }^{2 *}$ \\ ${ }^{1}$ Doctor in Medicine, Medical Assistant at Miami Dade Surgical Group, United States of America \\ ${ }^{2}$ Department of Ophthalmology, University of Medical Science, Cuba, Caribbean
}

*Corresponding author: Aldo A Sigler Villanueva, Department of Ophthalmology, University of Medical Science, Cuba, Caribbean

\section{Introduction}

According to Olsen (1978) a possible early description as a syndrome with retinal vascular tortuosity were reported by Beyer in 1958 [1]. Since that initial reference, several families with retinal arteriolar tortuosity, superficial macular hemorrhages and autosomal dominant transmission have been described worldwide [2]. Sutter \& Helbig (2003) made a review of this disturb [3]. In 2005 Plaisier et al. described a syndrome with retinal arteriolar tortuosity, haematuria and contractures with an autosomal dominant pattern and an evidence that is caused by heterozygous mutation in the COL4AI gene (120130) [4]. In the OMIM (Online Mendelian Inheritance in Man) data base, the Retinal Arterial, Tortuosity (RATOR) is accepted by an entry number 180000 [5]. We described the clinical characteristic of two sisters, 46 and 44 years-old, who had a retinal arteriolar tortuosity and several abnormalities such as brachydactylic and other dysmorphic disorders of fingers and toes, hallux valgus, telangiectasia in cheeks and upper thorax, protruding ears, frontal hyperostosis, pectus excavatum and mild mitral insufficiency. Their mother died and presented the same clinical features. The older sister shows in her right eye a best corrected visual acuity of hand movements. Anterior segment bio microscopy of her right eye was normal. From the gonioscopy view an open iridocorneal angle was seen. Ophthalmoscopy showed an abnormal tortuosity of retinal veins and arteries, damage and atrophy of the optic disk and hypopigmentation in the macular area (Figure 1). In her left eye there was no light perception. Band keratopathy and corneal oedema. Lens with cataract. The fundus red reflex is not visible and iris vascularization was observed (Figure 2). In an A/B scan, the anterior chamber was narrow. A fibrovascular band protruded from the optic disc into the vitreous. Neither vitreous opacities nor retinal detachment were noted. The other sister had the right eye enucleated more than 10 years before because of possible painful NVG. No histopathological report was found neither data in her clinical history. In her left eye the best corrected visual acuity was 0.1. Slit lamp examination revealed partial loss of pigment from the superficial layer of the iris. Gonioscopy exposed an open iridocorneal angle. Ophthalmoscopy examination showed an increase in tortuosity of the retinal veins and arteries, damage and atrophy of the optic disk where a capillary loop was seen (Figure 3). Both sisters had treatment with Timoftol $0.5 \%$ eye drops twice daily, by a previously elevated IOP. Doppler Ultrasound images of carotid arteries of the patients were normal. If you included all the clinical traits a presumable autosomal dominant disorder not previously described in view of its presence in two successive generations is suspected.

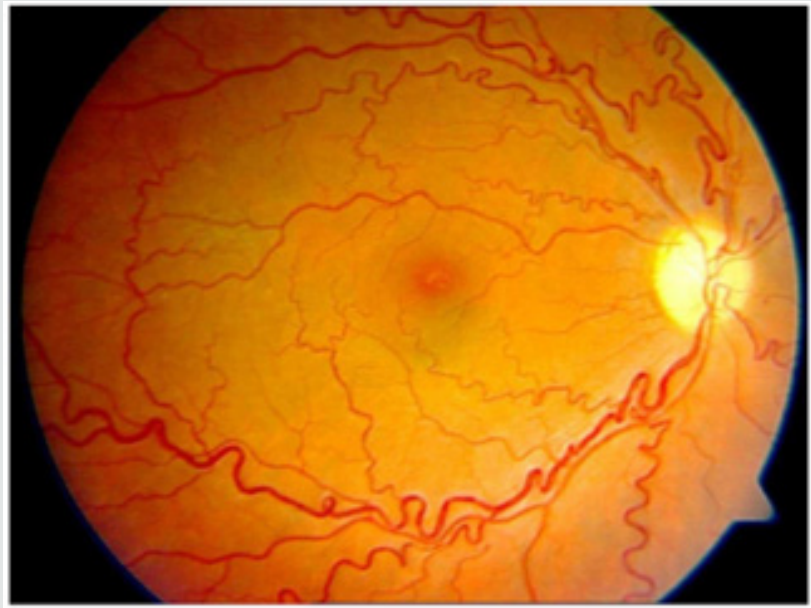

Figure 1: Fundus of right eye. The retinal arteries and veins are highly tortuous. The optic disk is pale and hypopigmentation in the fovea is seen. 


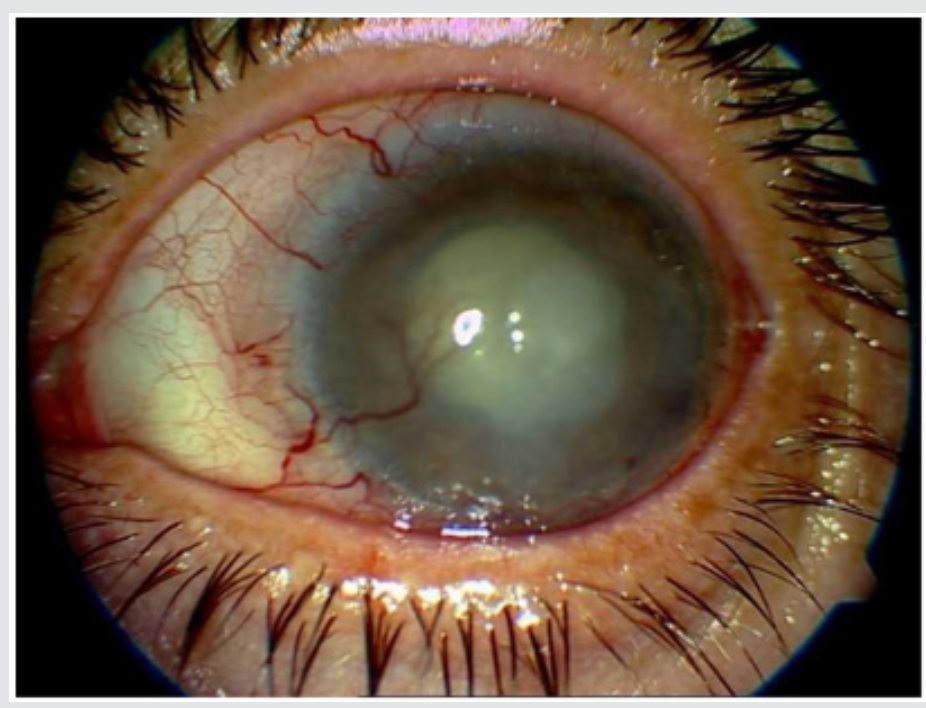

Figure 2: Left eye. Vascularization is seen in conjunctiva and cornea. Lens with cataract. Absolute glaucoma.

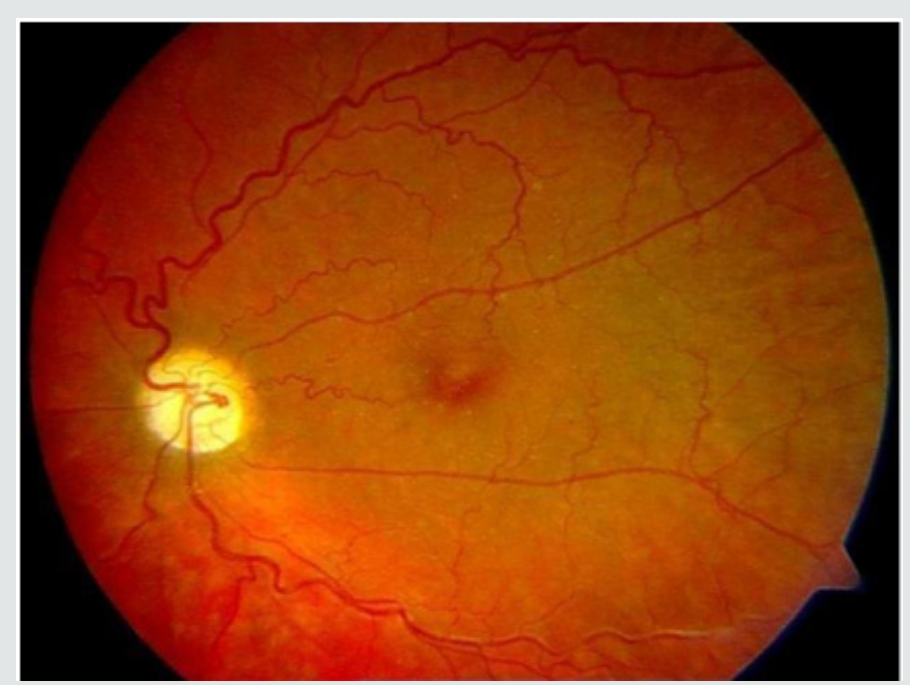

Figure 3: Patient IV-9. Fundus of left eye. The retinal arteries and veins show a certain degree of tortuosity. The optic disk is pale and excavated.

\section{References}

1. Olsen EG (1978) Retinal arteriolar tortuosity with retinal haemorrhage. A case report. Acta Ophthalmol 56(2): 322-325.

2. Sears J, Gilman J, Sternberg P (1998) Inherited Retinal Arteriolar Tortuosity with Retinal Hemorrhages. Arch Ophthalmol 116(9): 11851188.
3. Sutter FK, Helbig H (2003) Familial retinal arteriolar tortuosity: A review. Surv Ophthalmol 48(3): 245-255.

4. Plaisier E, Chen Z, Gekeler F, Benhassine S, Dahan K, et al. (2010) Novel COL4A1 mutations associated with HANAC syndrome: a role for triple helical CB3(IV) domain. Am J Med Genet 152A(10): 2550-2555.

5. Hamosh A, Scott AF, Amberger J, Valle D, McKusick VA (2000) Online Mendelian Inheritance in Man (OMIM). Hum Mutat 15(1): 57-61. 
CC) (P) This work is licensed under Creative Commons Attribution 4.0 License

To Submit Your Article Click Here:

Submit Article

DOI: 10.32474/RRHOAJ.2020.04.000195

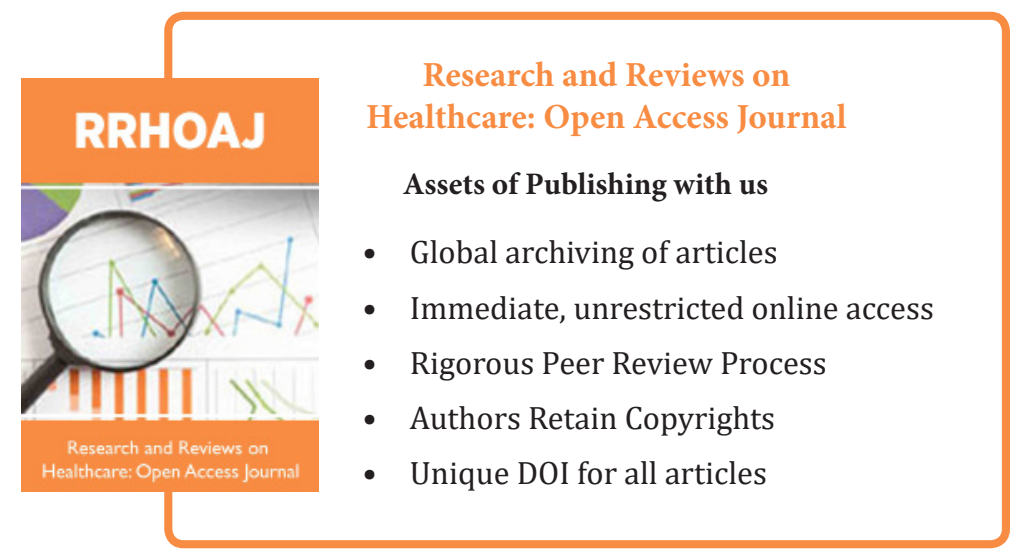

